\title{
Importance de l'étude de la répartition écologique pour la taxonomie des éponges calcaires
}

\author{
Radovan Borojević \\ Station Biologique, Roscoff, France \\ et \\ Laboratoire de Biologie des Invertébrés Marins, \\ Museum National d'Histoire Naturelle, Paris, France
}

\begin{abstract}
The importance of research on the ecological distribution for the taxonomy of calcareous sponges. The particular difficulties of the taxonomy of calcareous sponges are due to their great plasticity and their capacity for adaptation which obscure the natural limits of species. An attempt was made to define the natural populations of the Mediterranean species of the genera Clatbrina and Ascandra through a study of their ecological distributions. The populations not connected by the transitory forms were defined by their ecological preferences, and, as they are found in a close cohabitation, were treated as having the specific values. The variability of different morphological features is discussed, the features significant for taxonomy are pointed out and the morphologically close species are defined.
\end{abstract}

\section{INTRODUCTION}

Il y a une centaine d'années que la publication des travaux de Bowerbank et de Schmid ont marqué le début de l'histoire de la systématique des Éponges Calcaires, histoire que s'est déroulée depuis, comme c'est probablement le cas pour la plupart des taxonomies, en oscillant entre deux positions opposées. Plusieurs auteurs, notamment Brertfuss, Dendy et plus récemment Hôzawa et TANiTA, attribuent une valeur spécifique aux formes qui se distinguent par un ou par plusieurs caractères morphologiques. D'autres, comme Thacker et Topsent ont surtout porté leur attention sur la variabilité possible à l'intérieur d'une espèce, soulignant par exemple que certains spécimens peuvent être dépourvus d'une catégorie de spicules, ce qui a conduit SARÀ (1953) à réunir toutes les Leucosolenia et Clatbrina du Golfe de Naples en une seule espèce polymorphe; cette position a été abandonnée depuis par son auteur, mais elle est reprise par Bur Ton (1963) dans sa Révision de la systématique des Eponges Calcaires, où il réunit les 142 espèces du genre Leucosolenia citées par TANITA (1942) en deux espèces, et les 112 espèces des différents Sycon, Leuconia et Grantia sous le nom de Scypha ciliata. Bien que cette révision soit criticable à priori, car l'auteur ne tient pas compte des différences histologiques et ontogénétiques qui distinguent les nombreuses formes réunies dans ces espèces polymorphes, sa position est moins extrémiste qu'elle n'apparaît d'abord. La grande majorité des Éponges Calcaires, 
surtout celles du genre Clatbrina, ne se distinguent que par un seul des caractères du squelette. Si on accepte la variabilité de certains d'entre eux, ce qui est très probable, les limites entre espèces voisines deviennent très difficiles à préciser, ou, dans la plupart des cas, inexistantes.

Grâce à leur plasticité et à leur capacité d'adaptation aux conditions externes particulièrement développées, les éponges possèdent bien peu de caractères morphologiques stables: une étude comparée ou statistique, basée sur leur morphologie, ne peut donc pas nous aider à prendre position entre ces deux conceptions extrêmes. Nous avons donc essayé de préciser la distribution de chacune des formes qu'il était possible de distinguer parmi les Eponges Calcaires, et de définir ainsi les limites naturelles de leurs populations, pour pouvoir étudier ensuite la variabilité des caractères morphologiques à l'intérieur de chacune de ces populations définies par leur écologie.

\section{RÉSULTATS}

Nous donnerons ici l'exemple des espèces des genres Clathrina et Ascandra, étudiées en Yougoslavie le long de la côte dalmate, et en France dans la région de Marseille et dans celle de Roscoff.

En Méditerrannée, la distribution de ces éponges semble être surtout influencée par l'exposition de la localité aux vagues et à la lumière.

En ce qui concerne l'exposition aux vagues, C. cerebrum est la forme la mieux adaptée aux endroits très exposés; elle est suivie par $C$. primordialis, puis $C$. contorta, C. reticulum et $A$. falcata. Les formes $C$. clatbrus, C. coriacea et $C$. blanca sont communes aux endroits peu exposés.

D'autre part, parmi ces mêmes formes il existe une nette préférence pour l'exposition à la lumière. Ainsi C. cerebrum, C. primordialis et C. reticulum peuvent croître sur les endroits exposés au soleil, $C$. contorta et $C$. coriacea préfèrent les localités ombragées et $A$. falcata, C. clatbrus et C. rubra occupent les surplombs très ombragés et les grottes sous-marines.

En connaissant ces préférences, il est possible de prévoir exactement la distribution de chacune de ces formes: sur une surface réduite, toutes ces formes se succèdent et se remplacent mutuellement, selon l'exposition de l'endroit. Sur une paroi verticale, très exposée aux vagues, les enfoncements, les anfractuosités des rochers et les espaces entre les pieds des algues des premiers mètres au dessous du 0 sont occupés par $C$. cerebrum; déjà à 3-5 $\mathrm{m}$ apparaît $C$. primordialis, qui remplace $C$. cerebrum. Vers $10 \mathrm{~m}$ apparaît $C$. reticulum et $C$. contorta; dans les fissures profondes ou sous les surplombs se trouvent régulièrement $A$. falcata et C. rubra.

Dans les localités voisines, mais protégées, la distribution de ces formes montre un décalage vertical très net, de telle façon que C. cerebrum disparaît entièrement, remplacée par $C$. primordialis et $C$. reticulum; les grottes et les surplombs non exposés aux vagues sont riches en $C$. clathrus, $C$. coriacea et $C$. blanca, tandis qu' A. falcata y est absente.

Dans la région de Roscoff cette distribution est différente, car les marées introduisent des facteurs nouveaux. Une limite nette existe entre les formes intertidales, celles de la zone des Laminaires, et celles des parois plus profondes. D'autre part l'ap- 
port des sédiments côtiers définit la limite entre les éponges de la région côtière et celle de la zone du large.

La grande majorité des formes observées possèdent ainsi une distribution écologique très précise et constituent des populations bien délimitées. Dans la grande majorité des cas, ces populations ne sont pas reliées par des formes transitoires. Ce fait est inportant, car les populations citées se trouvent dans la nature suffisamment proches pour qu'y ait lieu, si celà était possible, une interfécondation: bien que sans preuve expérimentale, nos observations indiquent un isolement sexuel de ces populations, ce qui nous permet de leur attribuer la valeur de l'espèce.

Nous avons ainsi étudié et déterminé les caractères morphologiques communs à chacune de ces populations, significatifs pour la taxonomie. Mais nous avons dû conclure qu'aucun des types de caractères morphologiques n'est significatif dans tous les cas.

La présence ou l'absence d'un type particulier de spicules est p. ex. un caractère généralement valable. $C$. contorta et $C$. spinosa, qui se distinguent par la présence de diactines chez $C$. contorta, absents chez $C$. spinosa, représentent cependant deux extrémes d'une population variable, reliés par des formes transitoires: les spécimens les plus exposés à la lumière et aux vagues sont petits et munis de diactines, ceux de rochers à Halimeda tuna, moins exposés, en ont très peu ou en sont entièrement dépourvus. La morphologie des tétractines et des grands tubes exhalants rapproche pourtant ces deux formes. La forme d'un type de spicules peut aussi varier considéreblement; les tripodes typiques de C. cerebrum peuvent être très rares, ou ne différer des triactines normaux que par leur taille légèrement plus grande, ce qui est notamment le cas chez les spécimens relativement protégés.

D'autre part les différences morphologiques entre deux populations peuvent être très faibles, mais leur distribution indique qu'il s'agit d'espèces séparées. C. cancellata a par exemple une spiculation très proche de celle de la forme $C$. spinosa (qui appartient à la population de $C$. contorta), mais leur distribution est nettement différente: C. cancellata vit dans la région de Roscoff dans la communauté d'Axinella dissimilis et Pbakellia ventilabrum, tandis que C. spinosa est limitée à la région cotière, aux surplombs ombragés.

Ainsi les deux positions sont partiellement exactes, car les différentes formes morphologiques ont très souvent une écologie et donc une biologie différente, mais les variabilités intraspécifiques assez grandes sont possibles dans certains cas. Il est donc le plus souvent indispensable d'aborder les problèmes de la taxonomie des éponges par l'étude de leur écologie. En même temps une taxonomie précise peut fournir des informations très utiles à l'écologie générale, car les Eponges Calcaires, par leur électivité de leurs conditions de vie, semblent être d'excellents indicateurs écologiques.

\section{RÉSUMÉ}

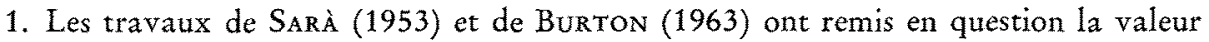
systématique des caractères morphologiques dans la classification des Eponges Calcaires, et ont proposé de considérer de nombreuses formes connues comme des va- 
riétés individuelles et régionales d'espèces très plastiques et variables. Nous avons essayé de préciser, par l'observation directe en plongée libre, la distribution et les préférences écologiques des Calcaires, de définir ainsi les limites naturelles de leurs populations, et d'étudier ensuite la variabilité des caractères morphologiques à l'intérieur de ces populations.

2. Nous exposons ici l'exemple des espèces des genres Clatbrina et Ascandra, étudiées en Adriatique, dans les régions de Marseille et de Roscoff. Leur distribution est en rapport direct et très précis avec l'exposition aux vagues et à la lumière, à tel point que sur une surface réduite plusieurs espèces se succèdent et se remplacent en fonction de l'exposition de l'endroit. Dans la règion de Roscoff cette distribution est plus large ce qui est dû aux effets des marées, mais les zones écologique préférentielles pour les espèces de Clathrina ont pu être établies en fonction de l'importance de l'apport des sédiments par les eaux côtières.

3. La possibilité de définir des populations dont la distribution est très précisemment délimitée malgré une coexistence dans un espace réduit, et qui ne sont pas réliées par les formes transitoires, permet d'éliminer l'hypothèse de la cospécificité de ces populations. Elle permet également d'établir les variations morphologiques possibles au sein d'une espèce, de retenir les caractères significatifs pour la classification, et finalement de distinguer les espèces qui se ressemblent morphologiquement. Dans le cas d'animaux qui, comme Clatbrina, possèdent très peu de caractères utilisables en systématique, c'est la seule façon de résoudre les problèmes de leur taxonomie.

\section{LITTÉRATURE CITÉE}

Burton, M., 1963. A revision of the classification of the calcareous sponges. Brit. Mus. (Nat. Hist.), London, $693 \mathrm{pp}$.

SARÀ, M., 1953. Variabilità delle Leucosolenie del Golfo di Napoli e nuove vedute sulla sistematica del gruppo. Annuar. Ist. Mus. Zool. Univ. Napoli 5, 1-110.

TAnita, S., 1942. Key to all the described species of the genus Letucosolenia and their distribution. Sci. Rep. Tôhoku Univ. (Ser. 4) 17, 71-93.

\section{Discussion suivant le rapport de BOROJEvić}

WEBB: Can breeding experiments be carried out on sponges?

Borojević: No breeding experiments were possible since spermatogenesis of Calcarea is unknown, and no "male" specimen were observed.

WEBB: Is evidence of relationship forthcoming from the subsequent aggregations of separated sponge cells?

BOROJEvić: Two series of experiments have given contrasting results: (1) Moscona and Humphreys described a neat specificity in reaggregation. (2) CURTIS and recently SARÀ have shown that mixed aggregations are possible, not only between different species of Sponges, but also between sponge and Cnidaria cells. 